\title{
THE FUNCTIONAL SIGNIFICANCE OF THE CARDIAC JELLY IN THE TUBULAR HEAR'T OF THE CHICK EMBRYO
}

\author{
ALEXANDER BARRY
}

Department of Anatomy, University of Michigan Medical School

TWO FIGURES

When, at about the 40th hour of incubation, the heart of the embryonic chick begins to pump blood it is essentially a simple tubular structure, looped to the right. Its wall consists of a tube of endothelium within a sleeve of epimyocardium. In living as well as in fixed preparations there is a space between these two layers. In the living condition this space appears optically homogeneous. In fixed preparations it is found to be filled with a delicate non-cellular meshwork of fibrils so fine as to be scarcely. visible under the oil immersion lens. Davis ('24) named this material cardiac jelly and demonstrated its gelatinous character in vivo. Its composition and structure in the living condition is still problematical. The present report is not primaily concerned with the composition of the cardiac jelly, but rather with its role in the functioning of the primitive tubular heart.

In the heart of a chick embryo of 40 hours incubation, the cardiac jelly surrounds the endothelium of that part of the heart which is destined to form the ventricle and truncus of later stages (Patten, '22). In cross section this sheath of cardiac jelly is not of equal thickness. It is distinctly thinner at the primary ventral and dorsal midlines. Cephalically it extends to the aortic roots, and caudally to the atrial region of the heart. In the fixed heart of a chick of this age, there 
is no marked regional thickening along the length of the cardiac tube. Thus, if the heart is to pump blood, the mechanism could not be other than the peristaltoid type of contraction, the nature of which is so clearly shown by the superimposed tracings from the Patten, Kramer films ('33). This means that the blood is propelled by a wave of contraction sweeping from the sino-atrial end of the heart to its truncus end. The presence of the relatively thick sheath of cardiac jelly might at first appear needlessly cumbersome in a heart of this type. However, an analysis of the mechanics of a peristaltoid beat makes it clear that the cardiac jelly is a requisite for effective pumping action.

To analyze the role of the cardiac jelly, let us assume a simplified tubular heart of circular cross section, and attempt to infer what its action would be if a layer of cardiac jelly were not present, that is to say, if the contractile sleeve of myocardium were in direct contact with the endothelium. If such a heart is to propel blood in a peristaltoid manner, then its lumen must be practically obliterated at systole. Strictly speaking a complete closure of the lumen would probably not be necessary, since the blood pumped is not a homogeneous fluid, but a suspension of cells. Thus, if the diameter of the lumen at systole were not appreciably larger than the diameter of a red blood cell $(20 \mu)$ it is likely that the corpuseles would pile up in the systolic constriction, and effectively prevent regurgitation.

Since in attempting the analysis of its action we are assuming that the heart is circular in cross section, we may let $R$ represent the radius of the myocardium, and $C$ its circumference. The subscripts $s$ and $d$ may be used to designate these dimensions in systole and diastole respectively. The myocardium of the embryonic heart behaves like most contractile tissues in that its systolic shortening does not exceed a certain proportion of its diastolic length. In living hearts of this stage of development studied in situ, this degree of shortening is approximately $20 \%$. The myocardium can be seen to shorten longitudinally as well as circumferentially, and will 
be assumed to do so to the same degree in the following analysis :

Thus,

$$
\mathrm{C}_{s}=.8 \mathrm{C}_{\mathrm{d}}
$$

hence

$2 \pi \mathrm{R}_{\mathrm{s}}=.8\left(2 \pi \mathbf{R}_{\mathrm{d}}\right)$

and,

$\mathrm{R}_{\mathrm{s}}=.8 \mathrm{R}_{\mathrm{d}}$

\begin{tabular}{|l|c|c|}
\hline \multirow{2}{*}{ Diastole } & Large Diameter & Small Diameter \\
\hline & \\
\hline
\end{tabular}

Fig. 1 Diagramatic cross sections of hypothetical tubular hearts without eardiae jelly in their walls. The two pairs of diagrams represent hearts of large and small diameters respectively. The systolic sections are drawn on the basis of a $20 \%$ shortening of the circumference on contraction. Note that when the diastolic diameter is large, the change in cross sectional area, (and hence in the stroke volume) is also large, but that the lumen does not close in systole. When the diastolic diameter is sufficiently small to allow systolic closure, the concomitant stroke is negligible.

From this it is clear that if the systolic radius of the lumen were to be only $20 \mu$, the diastolic radius would be $25 \mu$, and hence the heart would scarcely dilate on diastole, and could pump no significant amount of blood. Carrying this line of thinking a step farther, it is evident that in a tubular heart, the stroke volume will be determined, in part, by the diastolic radius of the lumen. Thus, if an appreciable quantity of blood 
is to be pumped, the diastolic radius must be relatively large, but if this is the case, the systolic lumen will be too large to permit adequate closure for pumping action.

It apppears, therefore, that if a tubular heart is to operate effectively, there are two requirements to be met. First, the radius of the epimyocardial sleeve must be relatively large if the heart is to have a significant stroke volume. Second, the lumen of the heart on systole must be nearly zero. A simple means of meeting this problem would be to allow the myocardium to be of relatively large radius, but to interpose between it and the endocardium a layer of material of such character that it would transmit the force of contraction radially down against the endothelium. This material would have to be firm enough to squeeze shut the lumen, and yet resilient enough so that it would not require an undue proportion of the energy of contraction in being so deformed. Obviously this layer could not be fluid in character, since if it were, the force of contraction would be transmitted along the entire length of the endothelial tube, and would cause no localized constriction. In the tubular heart of the young chick embryo we have exactly the type of construction suggested above, with a layer of resilient cardiac jelly lying between the epimyocardium and the endothelium.

Let us now modify the simplified tubular heart postulated above, by interposing a layer of cardiac jelly in its wall. By simple geometrical analysis we can make a rough estimate of how thick such a layer should be, if the heart were to function properly.

If we let $R$ be the radius of the myocardial sleeve, $r$ the radius of the lumen, and $t$ be the thickness of the cardiac jelly at diastole, then:

$$
\mathrm{R}_{\mathrm{d}}=\mathrm{r}_{\mathrm{d}}+\mathrm{t}
$$

Let us take a segment of this tubular heart of unit length at diastole. The volume of the cardiac jelly will be:

$$
\mathrm{V}_{\mathrm{d}}=\pi \mathrm{R}_{\mathrm{d}}{ }^{2}-\pi \mathrm{r}_{\mathrm{d}}{ }^{2}
$$


The volume of the cardiac jelly of this segment at systole, assuming that both the length and circumference of the myocardium have shortened to $80 \%$ of their diastolic value, will be:

$$
\mathrm{V}_{\mathrm{s}}=8 \pi \mathrm{R}_{\mathrm{s}}{ }^{2}-8 \pi \mathrm{r}_{\mathrm{s}}{ }^{2}
$$

As has been shown previously, the systolic radius $\left(\mathrm{r}_{\mathrm{s}}\right)$ requisite for an effective peristaltoid beat must be extremely small. For the sake of simplicity in formulation, this value $\left(\mathbf{r}_{\mathrm{s}}\right)$ will be taken as zero, since the error introduced by this assumption is negligible in the following analysis.

Therefore, if $\mathrm{r}_{\mathrm{s}}=0$

Then equation (5) becomes $V_{\mathrm{s}}=.8 \pi \mathrm{R}_{\mathrm{s}}{ }^{2}$

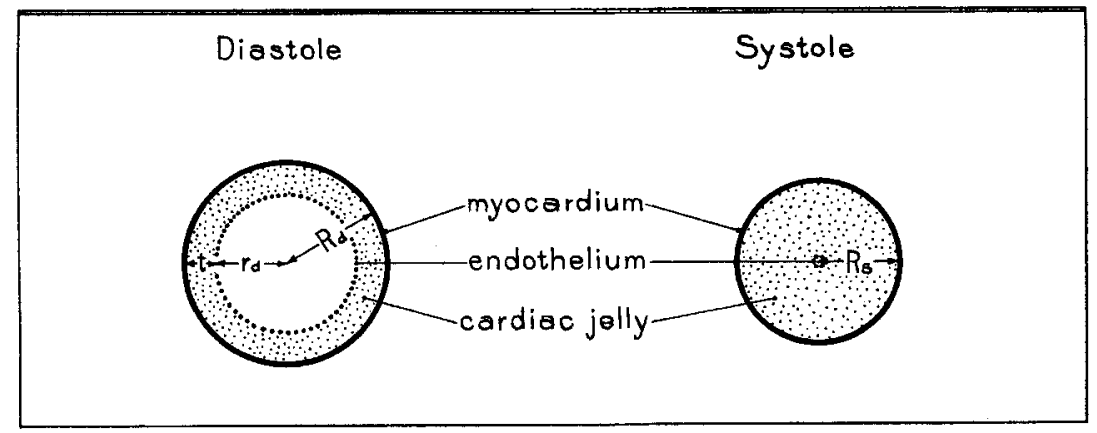

Fig. 2 Diagramatic cross sections of a tubular heart with cardiac jelly in its wall, showing its dimensions in the diastolic and the systolic condition. The diagrams are drawn on the basis of a $20 \%$ shortening of the cireumference on systole. The dotted line indicates the endothelium, the solid line, the myocardium, and the cardiac jelly is indicated by stippling.

$$
\begin{aligned}
t & =\text { thickness of the cardiac jelly layer in diastole } \\
\mathbf{r}_{\mathrm{d}} & =\text { radius of the lumen in diastole } \\
\mathbf{R}_{\mathrm{d}} & =\text { radius of the myocardial sleeeve in diastole } \\
\mathbf{R}_{\mathrm{s}} & =\text { radius of the myocardial sleeve in systole }
\end{aligned}
$$

If the cardiac jelly is essentially incompressible, then its systolic volume will be equal to its diastolic volume:

thus

$$
\pi\left(\mathrm{R}_{\mathrm{a}}{ }^{2}-\mathrm{r}_{\mathrm{i}}{ }^{2}\right)=.8 \pi \mathrm{R}_{\mathrm{r}}{ }^{2}
$$

However

$$
\mathrm{R}_{q^{2}}{ }^{2}-\mathrm{r}{ }^{2}=.8 \mathrm{R}^{2}{ }^{2}
$$

hence

$$
\mathrm{R}_{\mathrm{s}}=.8 \mathrm{R}_{\text {a }}
$$


and

$$
\mathbf{R}_{\mathrm{d}}{ }^{2}-\mathrm{r}_{\mathrm{d}}{ }^{2}=.8\left(.64 \mathrm{R}_{\mathrm{d}}{ }^{2}\right)
$$

(from equation 6)

hence

hence

Since

then

hence

hence

hence

or

$$
\mathrm{R}_{\mathrm{d}}{ }^{2}-\mathrm{r}_{\mathrm{d}}{ }^{2}=.512 \mathrm{R}_{\mathrm{d}}{ }^{2}
$$

$$
\begin{aligned}
r_{d}{ }^{2}=.488 R_{d}{ }^{2} & \sqrt{.488} R_{d}=.69 R_{d} \\
& R_{d}=r_{d}+t
\end{aligned}
$$

$$
r_{d}=.69\left(r_{d}+t\right)
$$

$$
\mathrm{r}_{\mathrm{d}}-.69 \mathrm{r}_{\mathrm{d}}=.69 \mathrm{t}
$$$$
-31 \mathrm{r}_{\mathrm{d}}=0.69 \mathrm{t}
$$$$
\mathrm{r}_{\mathrm{d}}=2.2 \mathrm{t}
$$$$
\mathrm{t}=0.45 \mathrm{r}_{\mathrm{d}}
$$

It is clear that in such a heart as we have been considering the deformation of the cardiac jelly will require a certain proportion of the energy of the myocardial contraction, and this portion cannot, therefore, be utilized in the propulsion of the blood. Such an apparent loss of energy seems to make the tubular heart an inefficient pump. However, the situation is not as wasteful as it appears. If we consider the status of the cardiac tube at the height of systole, we see the myocardium contracted, the layer of cardiac jelly deformed and squeezed down upon the endothelium so that the lumen is obliterated. When the myocardial sleeve relaxes during diastole, the layer of cardiac jelly, due to its own resiliency, springs back to its original shape, pulling the endothelium with it, and reestablishing a lumen in the heart. This reopening of the cardiac lumen well tend to suck blood into the heart. The blood will necessarily come from its venous end, since at the time of diastole of the atrial end of the tube, the truncus end is still in systole, effectively preventing regurgitation of blood from the aortic roots. Thus the energy of myocardial systole is in part used to force blood out of the heart into the arterial system, the rest being used to compress the resilient cardiac jelly, (i.e., being transformed into potential energy). This portion of the energy of each systole reappears during the subsequent diastole as mechanical energy in refilling the 
heart for the following systole. The tubular heart may be considered to be very similar in action to an aspirator bulb. When the bulb is squeezed, part of the energy is used to expel the contents under pressure, and part to deform the rubber of the bulb. When the force is relaxed, the bulb springs back to its original shape, its stored potential energy being released and used in refilling its lumen.

This hypothesis can be substantiated by direct observations. The tubular heart of a chick of about 40-48 hours incubation age observed in vivo, is sufficiently transparent to allow one to see the blood within its lumen. The contents of the lumen disappear on systole as the blood is forced out and the endothelial walls come into contact. If one transsects the body of such an embryo caudal to the sinus venosus, the venous return pressure to the heart will drop to the ambient pressure. Thus there is no pressure to force blood into the heart, and yet extravasated blood can be seen to be sucked into the heart during subsequent diastoles. This observation is understandable only on the basis of the sucking action produced by the heavy component of cardiac jelly in the wall of the heart at this stage of development.

By the 44th hour of incubation, the ventricular portion of the heart has been thrown into a loop, but the essential structure of its wall is unchanged. The truncus remains as a narrow tube, with a relatively heavy layer of cardiac jelly in its wall. Its lumen is rather narrow even in diastole. At this stage of development the fixed truneus does not have any localized thickenings of cardiac jelly along its length. Thus its entire wall must contract on systole sufficiently to close the lumen if it is to act in preventing regurgitation of blood into the ventricle. It must act in a manner comparable to that described above for the entire "primitive" tubular heart.

When the ventricle contracts, it ejects a gout of blood through the narrow truncus, dilating its lumen. One can deduce that some energy will be lost in this process. However, the truncus wall presents its minimal resistance to stretching at this phase, since the cardiac jelly of its wall is ensheathed 
by myocardium in diastole. As the blood passes through the truncus into the aortic arches, the pressure on the aortic side will increase. When the ventricle relaxes, its pressure drops to a low value. At this time the difference between the aortic and the ventricular pressures (reguritation pressure) will be maximal. This, then, will be the time when the valve action of the truncus is subjected to its greatest strain. However, it is precisely at this time, immediately after ventricular systole, that the contraction wave has swept to the truncus. Therefore its wall presents its greatest closing force, since its wall consists of cardiac jelly reinforced by myocardium in systole.

This type of valve action, although effective in preventing the regurgitation of blood, is relatively inefficient since its operation depends upon myocardial contraction, an energy consuming process. It also is inefficient in another respect. Since the ventricle must eject its blood through a narrow opening there must be considerable energy loss due to friction. Yet if the lumen of the truncus were larger, the contraction of its myocardial coat could not squeeze it shut against regurgitation.

In later developmental stages, when the endocardium of the truncus heaps up into endocardial cushion tissue pads, and as these pads gradually are molded to the definitive semilunar valves, a new mechanism of closure of the arterial outlet of the heart is introduced. By the time true value flaps are present, the ventricles empty themselves through a relatively large orifice, and the thin valves fold back against the wall of the outlet. When blood starts to regurgitate at the end of ventricular systole, the flaps are caught in the backflow, and swing across the lumen of the outlet, blocking it completely. This mechanism has two marked advantages over the primitive type of closure discussed above. First, the ventricle can empty itself through a reasonably large opening, thus reducing frictional losses. Second, regurgitation is prevented by the strength of the connective tissue of the valve flaps 
rather than by the contraction of myocardial tissue, and therefore, a considerable amount of muscular energy is saved.

The change from the first to the second type of valvular mechanism takes place gradually. During the intermediate stages one sees a progressive decrease in the myocardial functional component, and a corresponding increase in the connective tissue component of the truncus closure.

It is of interest to consider some of the developmental implications of the fact that in a tubular heart the thickness of the layer of cardiac jelly must be about $45 \%$ of the radius of the lumen in diastole if the heart is to propel blood with a peristaltoid beat. It is well known that the mass of the growing embryo increases rapidly, and it is axiomatic that the minute volume output of the heart must increase at a corresponding rate if the tissues are to receive an adequate blood supply. The minute volume of the heart is a product of the rate and the stroke volume. After the 50th hour the rate of the heart increases relatively slowly (Barry, '40). Therefore, much of this necessary increase in minute volume output must be accounted for by an increase in stroke volume. This requires a marked increase in the diastolic radius of the heart's lumen, which would necessitate a proportionate increase in the thickness of the cardiac jelly. It seems that if this were the case, an increasingly larger proportion of the energy of myocardial systole would be required to deform the cardiac jelly, and therefore less would be available for the propulsion of the blood. This situation is complicated by the fact that as the vascular bed of the embryo increases in extent, the aortic pressure must increase to maintain an adequate circulation through its increased resistance. Also as the diameter of the heart increases, the circumferential stress on its wall will increase in direct proportion even if the pressure remains constant. As a result of these two supplementary facts the entire wall must increase in strength (and therefore in thickness), to withstand the stress imposed upon it by its own greater diameter and by the greater systolic pressure. 
All of these factors lead one to predict that if the tubular heart with a peristaltoid beat were to persist as the heart of the developing embryo it would have to become progressively more massive and less efficient until it reached a stage at which it could no longer meet the requirements of the embryo. In the present state of our knowledge we cannot profitably attempt to compare the "efficiency" of embryonic with adult myocardium as contractile tissues. Nevertheless, it is of interest that the changes in the configuration and the arrangement of tissues in the embryonic heart are such that we see a marked increase in its efficiency as a pumping mechanism, even if we assume no increase in the physiological efficiency of the contractile component itself.

\section{LITERATURE CITED}

BARRr, A. 1940 Age changes in the pulsation frequency of the embryonic chick heart. Jour. Exp. Zool., 85: 157-170.

Davis, C. L. 1924 The cardiac jelly of the chick embryo. Anat. Rec., 27 : 201-202.

Patren, B. M., 1922 The formation of the cardiac loop in the chick. Am. J. Anat., 30: 373-397.

PATTEN, B. M., AND T. C. KRAMER 1933 The initiation of contraction in the embryonic ehick heart. Am. J. Anat., 53: 349-375. 